\title{
The Evolution of Users' Adoption Behavior under a Collaborative Service: An Agent-Based Computational Approach
}

\author{
Guoyin Jiang $^{*}$ \\ School of Information Management, Hubei University of Economics, Wuhan, 430205, China \\ School of Information Management, Wuhan University, Wuhan, 430073, China \\ Feicheng Ma \\ School of Information Management, Wuhan University, Wuhan, 430073, China
}

Received 26 May 2013

Accepted 16 January 2014

\begin{abstract}
As a relatively new collaborative service mode, Mobile Instant Messaging (MIM) is gaining ever greater popularity. MIM users interact within a complex system to influence others' adoption decisions, thus resulting in behavioral evolution. An agent-based computational approach is a methodology well suited to the simulation of this complex system. In this study, we build an agent-based computational model driven by empirical data. The model can present the evolution of the adoption of MIM. We also conduct simulation experiments to examine how some social and economic factors affect the behavioral evolution of the adoption of MIM. The results indicate that the average degree of nodes in a network has a significant effect on behavioral evolution in comparison with brand intensity. In contrast to the perceived usefulness, perceived ease of use and perceived entertainment value, the perceived cost and inter-operability has a lesser degree of influence on the adoption behavior. The different compositions of various groups have significantly different effects on the evolution of MIM adoption behavior.
\end{abstract}

Keywords: Collaborative service; Mobile Instant Messaging; Evolution; Agent-Based Computational approach

\section{Introduction}

Mobile Instant messaging (MIM) is a form of a realtime mobile communication service between two or more persons. MIM uses shared mobile software, through which clients can convey texts, pictures, documents, voice messages and videos. The advantages of MIM features such as mobility (access of network service at anytime, anywhere), portability and ease of use, also allow traditional instant messaging services to be migrated to a mobile platform. MIM is a popular collaborative mode (mobile platform and instant messaging tool), which is becoming a widely adopted individual communication tool, as well as an important application in the fields of e-commerce, mobile commerce and social commerce. Nowadays, more and more advanced mobile versions of once-traditional

*Corresponding author, Tel.: +86 2781972191

E-mail: guoyin_jiang@126.com 
instant messaging systems are being promoted on the Chinese market, such as Mobile QQ, WeiXin, Fetion and others.

Analysys International is a China-based consulting company, which reports that in 2009, there were 190 million MIM user accounts in China. By 2012, the number of user accounts had rapidly grown to 350 million, which amounts to 83.9 percent of all Chinese mobile phone users ${ }^{1}$. Mobile QQ, Mobile Fetion and Mobile MSN command the vast majority of the MIM market. Academic research on the applications of mobile instant messaging not only helps us better understand MIM services and its related consumer and organizational behaviors, but the research also supplies entrepreneurs with useful information which will help them make decisions on how to increase their market share of mobile instant messaging products.

Published studies exist which examine the adoption or acceptance behavior of MIM users, but these studies do not generally focus on the transition from using an instant messaging service to using an MIM service. In the transition process, user behavior is a dynamic evolutionary procedure composed of numerous stages, some of which exhibit complex phenomenon. To resolve the evolution pattern in this process, we employ the Agent-Based Computational Economics (ACE) approach driven by empirical data. The ACE is a computational study of economic processes modeled as dynamic systems of interacting agents, and can be used in research fields of complex systems ${ }^{2}$.

Agent-based computational method (ACM ) has been used to explore the adoption and diffusion of new products and other complex systems ${ }^{3}$. Each agent in an ACM represents one user or consumer, and the links represent the connections between users. Each agent's behavior is determined by decision-making rules ${ }^{4}$. Our research uses agents to represent the users of IM, in such a way that ACM as a whole can describe the interaction among agents under different degrees of nodes in a network, such as brand intensity, ease of use, usefulness, entertainment value and inter-operability costs. Subjective norms change over time, and these determinants may impact on users' adoption of IM or MIM. Our results demonstrate how intrinsic and extrinsic motivations affect the evolutionary adoption of MIM groups. The research results can help MIM vendors decide how to enlarge their share of the MIM market.

The structure of this paper is as follows: The next section will review the related problems of MIM. Section 3 briefly introduces the empirical model and it's results. This is followed by a description of the agentbased model and simulation system in section 4 . The simulation results and discussion are contained in sections 5 and 6 . Finally, section 7 presents the study's potential contributions and implications.

\section{Literature review}

An instant messaging (IM) user makes the decision of whether or not to adopt a mobile instant messaging (MIM) service, based on his or her perceived social or environmental influences. Because many consumers are already members of existing social networks, their decisions can be affected by family members, friends, workmates, managers and other users in the social network. An experimental study is employed to examine how perceived technical characteristics and other social influence factors impact on the evolution of MIM users' adoption decisions.

\subsection{Empirical research of IM/MIM}

Classical and empirical theories include the Theory of Reasoned Action (TRA) ${ }^{5}$, the Theory of Planned Behavior (TPB) ${ }^{6}$ and the Technology Adoption Model (TAM) ${ }^{7}$. Similar to the adoption of IS/IT, the adoption of IM/MIM is a class of complex problem related to consumer behavior. Drawing upon the TRA, TAM, TPB and other theories of social psychology, many researchers have empirically examined how factors affect the acceptance behavior of IM users ${ }^{8-11}$.

MIM has wide and growing applications since the introduction of advanced mobile equipment and $3 \mathrm{G}$ technology. However, few academic studies focus on users transferring from IM to MIM. From the perspective of service management, Deng et al. have investigated large samples to demonstrate that trust, service quality, functional value and emotional value have significantly affected the degree of Chinese MIM users' satisfaction and loyalty ${ }^{12}$. Jiang et al. have empirically explored how various factors impact on users' adoption of Mobile Instant Messaging (MIM) in China, based on the Technology Acceptance Model (TAM) and the social network theory. Their research results show that social and technical factors significantly affect the adoption of $\mathrm{MIM}^{13}$.

\subsection{Evolution of IM/MIM}

ACM can represent micro interaction between agents, and further deduced macro emergence rules. ACM is used to simulate a complex system. Ma et al. used a multi-agent to construct an NK model of multiple vendors, multiple products and multiple consumers to analyze the evolutionary process of technology diffusion $^{14}$. Considering consumer preferences and the 
agents' levels of learning, Koentakes constructs a model of client loyalty to analyze users' purchasing behavior ${ }^{15}$. Jiang et al. employ an agent and discrete simulation to explore the evolution of interactive behavior based on matching roles and dynamic tasks ${ }^{16}$. In these studies, the interaction between agents is based on matching methods and learning theories, but validation is obtained without any actual data. In our study, we build a learning function driven by empirical data, which takes into consideration the matching of characteristics between agents.

Network effects and network topology both have effects on the diffusion process ${ }^{17}$. Network effects and diffusion channels have been recognized as important factors in the evolution of agents ${ }^{18}$. In light of how consumers interact, a consumer group can be regarded as a network, which can, for example, be in the form of an office network or a friendship network. These networks are classified as complex and are characterized as classical complex network structures, similar to small-world, scale-free or random networks ${ }^{19}$. With different network frameworks, the effects of transmission between consumers are different. Yu et al. use a small-world network to simulate the implicit diffusion process of knowledge within an entity. They put forward three strategies as means to learn from individual consumers, and conclude that the accommodation inclined learning strategy is the most effective means to diffuse implicit knowledge among an entity $^{20}$. Zaffar finds the interaction effects between network topology, network density and inter-operability costs strongly influence the diffusion dynamics of an OSS (Open Source System), based on the Agent-based Computational Economics (ACE) approach ${ }^{21}$. Schramm introduces brand factors, such as features, pricing, promotion and distribution into ABM; the simulation of output represents the different phases of the diffusion curve, such as the initial or takeoff phases ${ }^{22}$. In this study, we use a preferential attachment network, which is somewhat similar to a scale-free network, and bearing in mind that some real social networks are scale-free networks ${ }^{23}$.

\section{Empirical model and data analysis}

In this paper, we employ an agent-based computational approach to explore the evolutionary discipline of mobile users' behavior. The agent-based model is driven by empirical data. We briefly introduce an empirical model and demonstrate the methodology used in this section.

\subsection{Empirical model}

From previously published studies, we find that user adoption behavior is affected by the perceived ease of use (PEOU), perceived usefulness (PU), subjective norms (SN) and other factors in IM and MIM empirical adoption models. Drawing upon a classic TAM model, we construct an advanced or modified TAM model. Figure 1 shows the empirical model which was proposed in prior research (Jiang et al., 2011) ${ }^{13}$. Then, we examine some of the technical and social attributes that affect a user's adoption of MIM. A technical attribute is an individual's perception of the function and performance of information systems or technology. Social factors such as synergy value and social norms have an indirect effect on people's behavior. In other words, people learn from or draw a certain amount of knowledge from colleagues, friends, relatives or other people who have already perceived the value of information systems or technology.

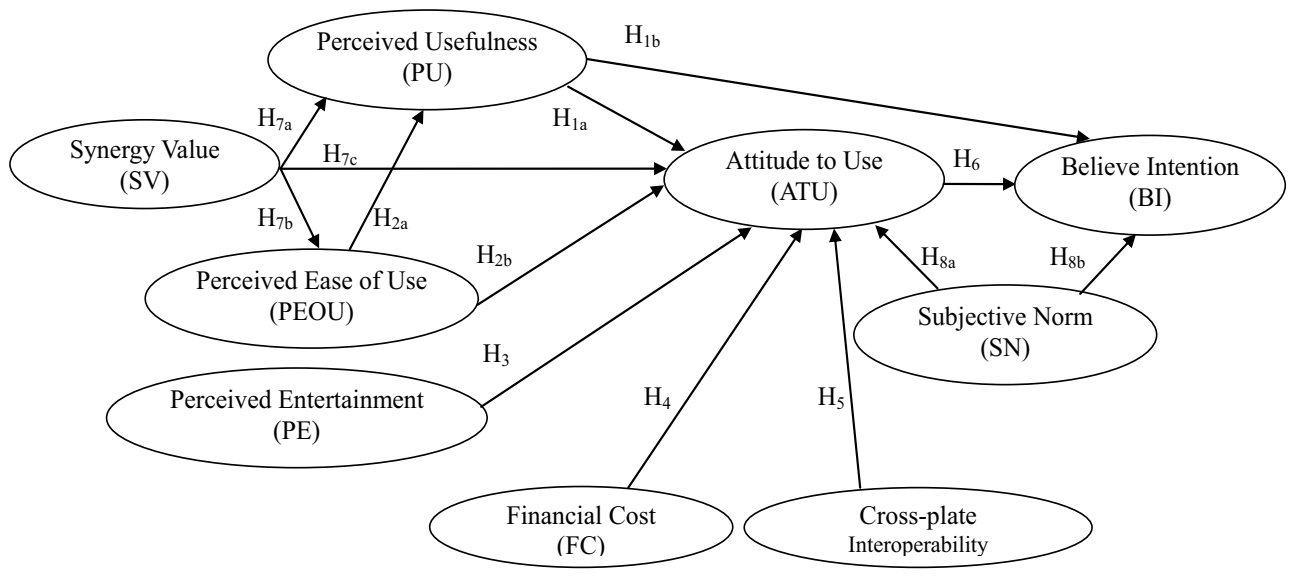

Fig.1. Empirical model ${ }^{13}$ 


\subsection{Empirical methodology and analysis results}

We conduct survey data by means of SPSS and Amos 7.0 to test the empirical model. The detailed methodology and data analysis are represented in our prior work (see reference [13]). The same data sample and method employed in our prior work are used to empirically test the model by grouping, and the standardized path coefficients of MIM are shown in Fig. 2. Based on these path coefficients, we can compute the value of the belief function of agents in our computational system.

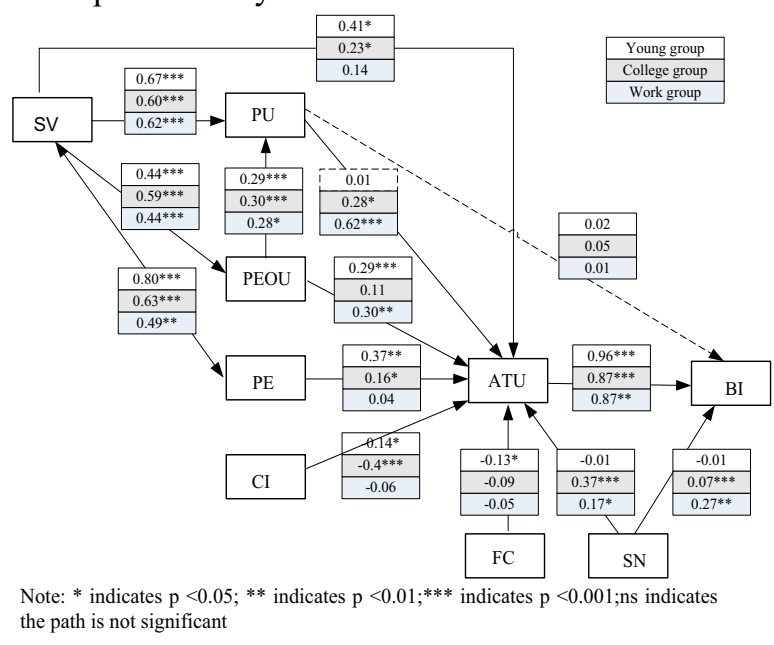

Fig. 2. Empirical model for groups

\section{Computational methodology}

The agent-based computational modeling (ACM) method is more suitable for modeling dynamic systems and other complex phenomena than are other approaches. Our study uses a computational simulation system to visualize the evolution of MIM adoption, and we explore how economic and social factors effect the evolution of MIM adoption.

\subsection{Empirical methodology and analysis results}

We build a computational model based on an agent and social network. An agent represents one person who is a user of an instant messaging tool. The logic inference process of an agent's decision is based upon the path coefficient in Fig. 2.
Definition 1: We define $b e_{i}(t)$ as an agent's increased belief at time $t$, which is a degree of the agent's intention to adopt mobile instant messaging. This degree is equal to other factors affecting the belief intention of agent $i$ in the structure model, which includes both direct and indirect effects.

$$
\begin{aligned}
& b e(t)=(p s v(t)-p s v(t-1)) * \\
& \text { psv_puse* puse_bi+(psv(t) - } \\
& p s v(t-1)) * p s v \_p u * p u \_a t u * \\
& a t u \_b i+(p s v(t)-p s v(t-1)) * \\
& p s v \_p e o u * \text { peou_atu* } \\
& a t u_{-} b i+(p s v(t)-p s v(t-1)) * \\
& p s v \_p e o u * \text { peou_pu }{ }^{*} p u_{-} b i+ \\
& (p s v(t)-p s v(t-1)) * p s v \_ \text {peou } * \\
& \text { peou_pu* pu_atu*atu_bi+ } \\
& \text { usefulness }(t) * p u \_b i+ \\
& \text { usefulness }(t) * \text { pu_atu* } \\
& a t u \_b i+\text { easeofuse }(t) * \\
& \text { peou_atu*atu_bi+ } \\
& \text { easeofuse }(t) * \text { peou_pu* } \\
& \text { puse_bi+easeofuse }(t) * \\
& \text { peou_pu* pu_atu*atu_bi+ } \\
& \text { entertainment }(t) * \text { pe_atu* } \\
& a t u \_b i+i n t e r o p e r a b i l i t y(t) * \\
& c i \_a t u * a t u \_b i+\operatorname{cost}(t) * \\
& f c \_a t u * a t u \_b i+\varepsilon
\end{aligned}
$$

Where, $\varepsilon$ is a residual value, which is the residual of $B I, p s v \_$puse is the path coefficient from $p s v$ to puse in Fig. 2. The naming rule of puse_bi, psv_pu, pu_atu, atu_bi, psv_peou, peou_atu, peou_pu, pu_bi, pu_atu, atu_bi, ci_atu, and $f c \_a t u$ is the same as $p s v \_$puse.

Prior literatures show that network externalities existed in consumer networks ${ }^{24}$ or other social networks $^{25}$. That is, one benefits from other adopters in the network. Because word of mouth or other social norms have an effect on present values $^{24 ; 26}$, to quantify the value of the benefit of each agent under network externalities, we give Co-published by Atlantis Press and Taylor \& Francis Copyright: the authors 
$p s v(t)$ as the benefit from other adopter agents in the network at time $t$.

Definition 2. The $p s v$ is the synergy value of node $i$. That is, agent $i$ can perceive the value of network externalities. The value is equal to the square of the ration of the adopted user and total users.

$$
\operatorname{psv}(t)=(\operatorname{an}(t) / d n(t))^{2}
$$

Here, the $a n(t)$ is the adoption number of mobile instant messaging within neighbors of agent $i$ at time $t$, and the $d n(t)$ is the total number of neighbors of agent $i$ at time $t$.

In formulation 1, the parameters are represented in table 1 , with the exception of $p s v$. The value of $p s v$ puse, $p s v \_p$ and other coefficients can be found in the path coefficients of Fig. 2 .

The interaction relationship among different agents is based on Definition 3.

Definition 3. Denote the matching degree among different agents, which is the degree of one agent's imitation of his or her neighbor agents.

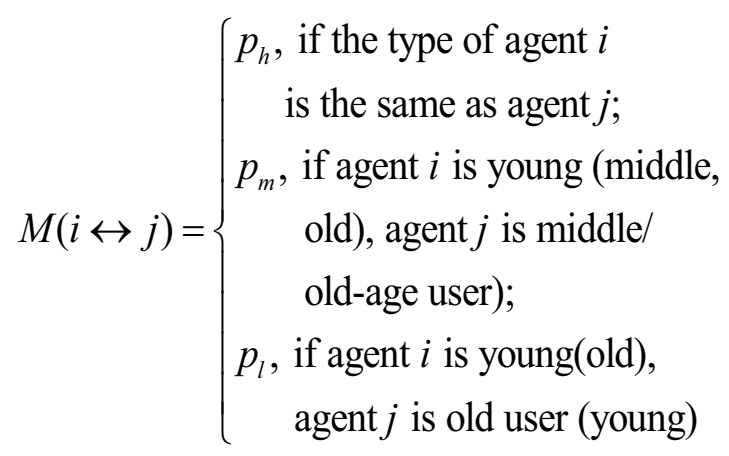

Here, $i, j$ is the identifier of the agent; $p_{h}, p_{m}$ and $p_{l}$ are the probabilities which are subject to uniform distribution, and $p_{h} \geq p_{m} \geq p_{l}$.

\subsection{Introduction to NetLogo}

Based on the computational model proposed above, we used the Logo language to implement the computational model based on NetLogo. NetLogo is a programmable system used to model and simulate social phenomena platforms.
NetLogo's modelers can give instructions to hundreds or even thousands of "agents" all operating independently. According to the connections between the behaviors of individuals at a micro level, some rules for the interaction between agents can be used to build an agentbased model, which is then used to explore the macro-level patterns that emerge from the interaction between many individuals over a given period of time.

\subsection{System User Interface}

The experimental system based on NetLogo is responsible for scheduling and arranging the simulation ticks. The user interface (UI) for the evolution of the adoption of MIM based on NetLogo is given in Figure 3.

The UI includes four sections:

(1) The system menu is located above and includes "file," "edit," "tools" and "other items." A user can build a simulation system based on the models library. The system can be run on ticks or on a continuous operation basis. Run speeds can be set at lower, normal and faster speeds.

(2) Simulation results at every tick are displayed at the lower left. The results include the changes in the counts of instant messaging, mobile instant messaging and mobile instant messaging resistant users.

(3) The agent's evolution on the network is displayed at the lower middle corner. Agents have three statuses, one of which is a status of using instant messaging, which is represented by a green colored node. The second is a status of using mobile instant messaging, which is represented by a red colored node, and the third status is that of an agent who is resistant to mobile instant messaging. That status is represented by a gray colored node.

(4) At the lower left corner is the parameter interface. We can adjust the use of parameters to view changes in evolution and to collect the related data. Default values for the parameters are shown in Table 1. 


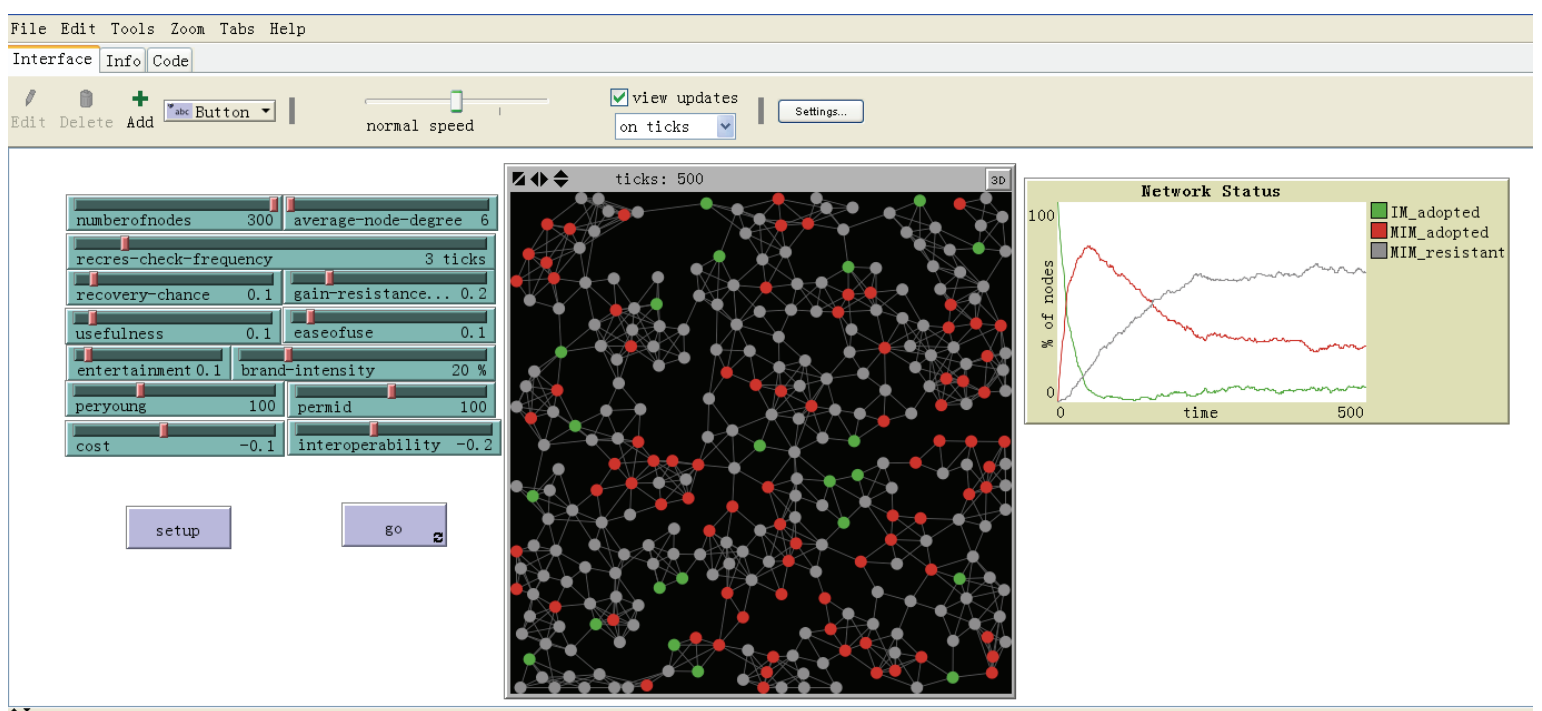

Fig.3. User interface (UI) for the evolution of MIM adoption

Table 1. Default values for Parameters

\begin{tabular}{cllc}
\hline No & \multicolumn{1}{c}{ Parameter } & \multicolumn{1}{c}{ Description } & Value \\
\hline 1 & Number of nodes & The number of users & 300 \\
2 & average-node-degree & Average node degree of user network & 6 \\
3 & usefulness & Usefulness of mobile instant messaging (i.e. usefulness in formulation 1) & 0 \\
4 & easeofuse & Ease of use of mobile instant messaging (i.e. easeofuse in formulation 1) & 0 \\
5 & entertainment & Entertainment value of mobile instant messaging (i.e. entertainment in & 0 \\
6 & brand-intensity & formulation 1) & Brand intensity of mobile instant messaging \\
7 & peryoung & Percentage of young users among total group & 20 percent \\
8 & permid & Percentage of middle-aged users among total group & 0 \\
9 & cost & Cost increases for mobile instant messaging (i.e. cost in formulation 1) & 0 \\
10 & interoperability & Interaction of mobile instant messaging (i.e. interaction in formulation 1) & 0 \\
\hline
\end{tabular}

\section{Simulation and result analysis}

We conducted the experiments by adjusting parameters as shown in Table 1. Under each parameter combination, we can collect simulation data relating to the evolution of users' behavior by running an agent-based computational system. Each scenario must run for 500 ticks.

\subsection{Effects of the average degree of nodes in a network and the brand intensity of products}

Prior research has shown that the topology structure of a network affects the purchasing decisions of consumers from a social network perspective, in much the same way as does the brand intensity of the product. The degree of a present network's structure is a key indicator for its evolution. In this section, we conduct experiments to test the sensitivity to the evolutionary process when the parameter is increased by 1 percent, the degrees of nodes are from 6 to 15 when the brand intensity of a product is 20 percent, and when the brand intensity of products are from 20 percent to 23 percent when the degree of nodes is 6 .

From Fig. 4a, we can see that with an increase in the degree of a node, the maximal adoption number of MIM increased, i.e. the degree of the node has a positive correlation with the maximal number of an adopter. When the degree of a node is 6 and the brand intensity of a product is 20 percent, with an increase in degree of from 6 to 15, respectively, the maximal numbers of adopters are 75.33, 84.7, 91.7 and 94.67. From Fig. 4b, we can see that the maximal numbers of adopters are $75.33,69.33,74.7,80.3$ and 78.3 , with an increase in brand intensity from 20 percent to 23 percent, but we can find no positive evidence of a correlation between maximal adopters and the brand intensity of a product. Therefore, we can conclude that the degree of a node has a more significant effect on behavioral evolution than does brand intensity. It is evident that building direct links between nodes can help speed up diffusion. Managers should therefore add new diffusion channels 
or advance old diffusion channels. This strategy is more effective than trying to increase brand intensity through advertisements or other promotional strategies.

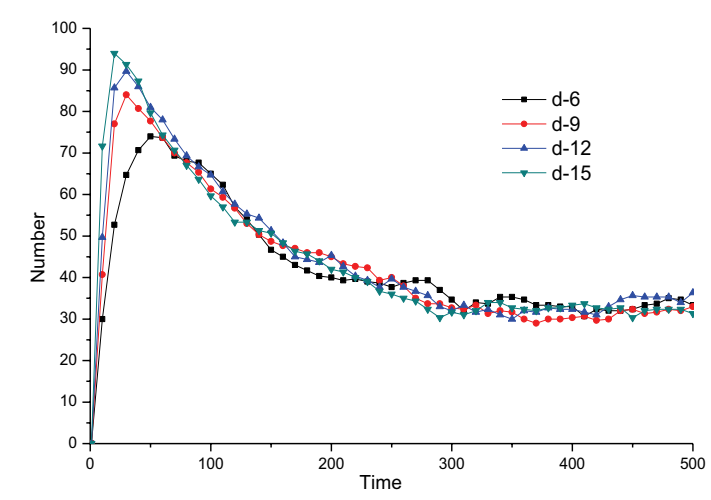

a. effect of average node degree of network

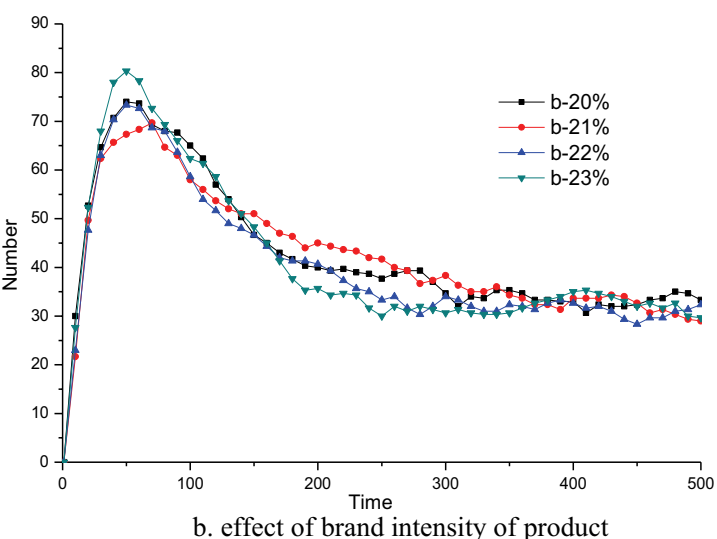

b. effect of brand intensity of product

Fig. 4. The effect of the average node degree of a network and brand intensity of a product

\subsection{Main effect of parameters in an empirical model}

Empirical analysis shows that perceived usefulness has a significant effect on a user's attitude towards the use of MIM, as do perceived entertainment, cross-platform interaction and perceived ease of use. However, crossplatform OS and costs have a negative correlation with an individual's attitude.

Adoption and diffusion processes take on several stages over a given time. From Fig. 5a to 5h, each evolution curve can have several main stages, including production, promotion, maturity and recession stages. It is evident that all evolution curves can be divided into three stages. The first evolution stage is the promotion and growth period, which occurs at approximately the times from 1 to 35 . The second stage is the maturity period, which occurs at approximately the times from 36 to 100 . The remaining time is the recession period.

From Fig. 5, we can observe how different parameters have various effects on the behavior of the user group. At different stages, adopters exhibit different behaviors, and different service characteristics have significantly different effects on the diffusion process. User groups represent the differences in diffusion behavior. The analysis reveals that the perceived usefulness, perceived ease of use, perceived entertainment value, and the cost and inter-operability factors have significant effects on behavioral evolution.

Statistical results show that increases in parameter values can significantly affect the evolution of behavior, i.e. the behavioral evolution will correlate with the perceived usefulness, perceived ease of use and the perceived entertainment value of MIM, but the associated costs and inter-operability factors have a negative correlation with behavioral evolution during the early stages.

Generally speaking, statistical methods are applied to gain potentially valuable knowledge from a large number of data samples. The precondition of common statistical models requires that test samples follow normal, Poisson or other common distribution channels. In this study, we explore One-sample KolmogorovSmirnow ${ }^{27}$ to test the distribution of data samples. The results indicate that data samples are not subject to the common distribution methods, such as normal, uniform, Poisson, and exponential distribution ( $p$ value $=0.000<0.05)$. Therefore, it is not possible to use many common statistical methods based on common distribution methods to test data samples. Nonparametric methods have been developed in cases where the researcher knows nothing about the parameters of the variable.

In this study, the $K$ Independent Samples method is used to test the effects of the varied parameters. This method is a non-parametric statistic method used to test for differences in samples. We test the distribution of simulation data in different parameters by SPSS. The analysis results indicate significant differences in data samples under different parameter settings ( $p$-value < $0.05)$.

During the production and promotion stage, the factors of perceived usefulness, perceived ease of use and perceived entertainment value have a positive correlation with adopter numbers, but these parameters have a less obvious negative correlation with adopter numbers over time.

To compare the variations of each parameter's effect on the number of adopters, we represent data in figures 
with the same conditions (Fig. 5f-5h). Overall, the factors of perceived cost and inter-operability have less of an impact on the number of adopters than do the factors of perceived usefulness, perceived ease of use, and perceived entertainment value. Thus, product characteristics such as usefulness, ease of use, etc., are the user's main concerns when entering the market. These factors determine the adoption behavioral decision of whether or not they purchase. After considering the essential product characteristics, users will focus on the associated costs and inter-operability. This knowledge will provide managers a number of implications when making strategy decisions on the promotion of their product in the marketplace. For example, the intrinsic properties of a product will significantly affect its entry level in the market. Therefore, vendors should improve as much as possible the product's characteristics at the earliest possible product diffusion stage, and then adopt their promotional strategies at a later stage. With the introduction of other homogeneous, competitive products and the deepening of users' experiences, potential customers will, over time, care about the interoperation and cost factors to the same degree as they care about the other product characteristics. Form Fig. $5 \mathrm{~g}$ to Fig. $5 \mathrm{~h}$, we find that the variations in adopter numbers become smaller with increases in parameters.

\subsection{Effect of the group component}

The user group consists of young and middle-aged college students, as well as workers. In this paper, we test how different groups impact on behavioral evolution. We collected the data of four group combinations, which include $(50,50,200),(50,200,50)$, $(100,100,100)$ and $(200,50,50)$. Each component of a group combination consists of three parts. The first part is the number of the younger members in the group; the second is the number of college students, and the third part is the number of workers. For example, the combination $(50,50,200)$ represents a group which has 50 younger people, 50 college students and 200 workers.

We conduct data sampling by using the $K$ Independent Samples method in SPSS. The results show that the factors of perceived usefulness, perceived ease of use, perceived entertainment value, perceived cost and inter-operability have different effects on behavioral evolution under the four groups' samples ( $p$-value $<0.05$ ). When usefulness increases, groups can diffuse information quickly, and older members dominate groups in the early stages. With the percentage of other kinds of groups increasing, the diffusion process becomes slower. When one group member type absolutely dominates the group, the information diffusion is the slowest. It is significant that usefulness is the key factor for older groups, who are the most rational consumers, and who mind whether or not the MIM can help them with their work. However, younger members do not care as much about the MIM's usefulness.

We also find that the ease of use of MIM product has a greater effect on the behavior of younger groups than on that of elders. Compared with elders' attitudes to the perceived entertainment value, younger groups and college students' attitudes dominated the group. This is because younger people are apparently more sensitive to the entertainment value of a product. The early diffusion stage is faster in groups dominated by younger people and college students. Therefore, increased entertainment characteristics will be more attractive to younger groups than to elderly groups. Promoting the entertainment value of an MIM product can help the product to penetrate a market more quickly when younger groups dominate that market.

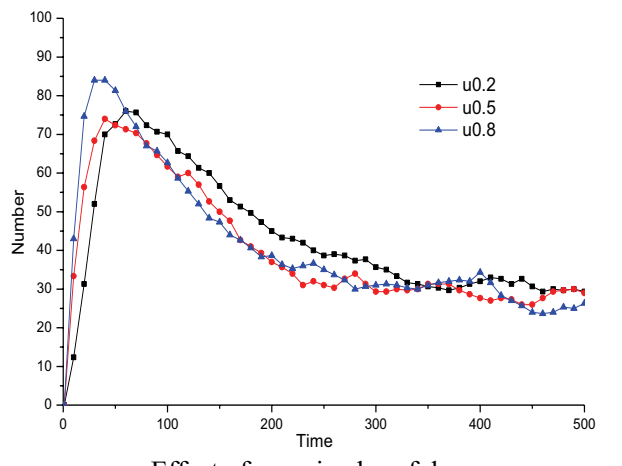

a. Effect of perceived usefulness

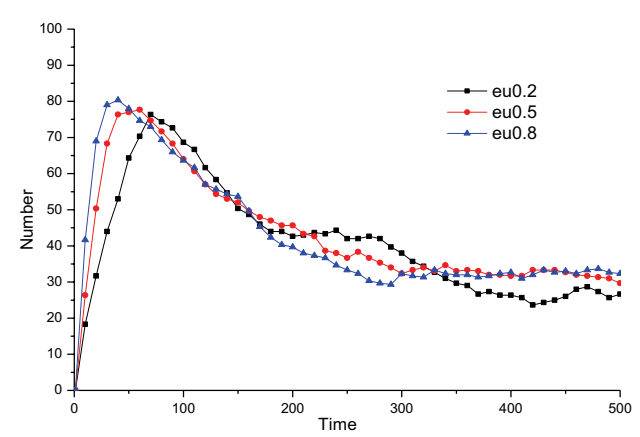

b. Effect of perceived ease of use 

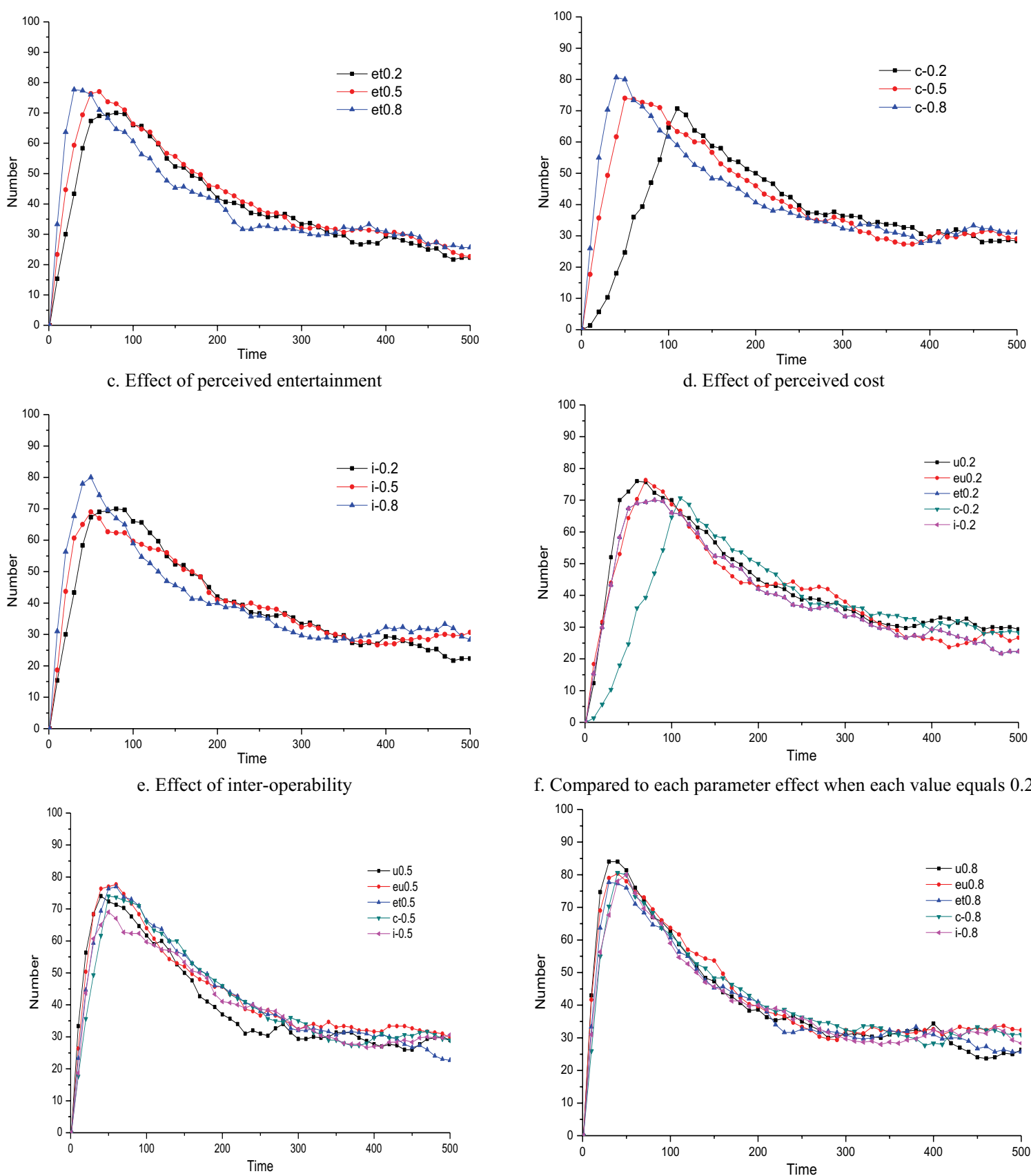

f. Compared to each parameter effect when each value equals 0.2

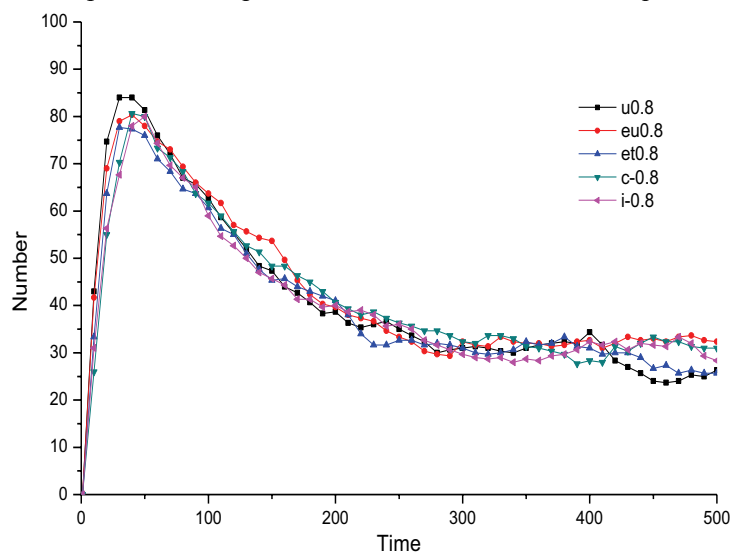

g. Compared to each parameter effect when each value equals $0.5 \quad$ h. Compared to each parameter effect when each value equals 0.8

Fig.5. The effect of parameters in an empirical model

\section{Discussions}

The objectives of this paper can be condensed into three parts: a) Construct a model for the investigation of the technical and social factors affecting the adoption of MIM, and empirically examine how technical and social factors affect the adoption of MIM in different groups; b)
Build an agent-based computational model driven by empirical data to simulate the evolution of a group's MIM adoption behavior; c) Explore the effects of the factors of perceived usefulness, perceived ease of use, perceived entertainment value, cross-platform interaction and the associated costs of MIM adopters on their behavioral evolution. 


\subsection{Contribution to research}

This study has made the following contributions to the growing body of research on behavioral evolution. It has provided a research perspective through a simulation study of behavioral evolution driven by empirical data. In order to increase the validity of our simulation, we constructed an empirical model drawing on existing published studies, and we used data obtained from a questionnaire to examine path coefficients. On the basis of these path coefficients, we built a computational model for the evolution of the adoption and use of MIM. The computational model has more reasonable parameter settings than some traditional simulation models, and the simulation results are more credible and reliable.

Furthermore, this research focuses on group evolution processes from a perspective of multi-dimensional comparisons. Our study not only considers how intrinsic and extrinsic factors impact on evolutionary behavior, but also considers the sensitivity of each variable within the study groups at different levels, and the changes of diffusion speeds at different time periods.

\subsection{Contribution to practice}

Our research can provide MIM vendors numerous implicative suggestions and can also provide some system support. Overall, the simulation results demonstrate that the evolution of a product can be influenced by different parameters. With increases in usefulness, ease of use and entertainment value, and a decrease in associated costs and inter-operation difficulties, the diffusion process into the marketplace can become faster. Compared with the cost and interoperation characteristics of a product, the perceived usefulness, ease of use and entertainment value all have a significantly greater effect on the evolution of adoption behavior in the early stages. In different groups which are composed of different proportions of younger people, college students and workers, each promoted policy has a different effect on each class of the group's members. These findings can support the decisions made by a product's R\&D and marketing managers at different stages of the product's development in terms of the relative importance of the product's intrinsic technical characteristics compared to other factors, such as the cost of use, entertainment value, etc. Compared to related published studies, our simulation model provides a richer and clearer depiction of the critical variables and their interactions with each other under multi-period settings. An agent-based model driven by empirical data can empirically examine dynamic consumer behavior and bridge the gap between the empirical research based on static data (which is often used as fields of static and limited dynamic behavior) and traditional simulations based on assumed input. The current computational model gives an example of how to incorporate the two methods in one model to simulate a complex system.

\section{Conclusion, limitation and future research}

In this paper, we build an acceptance model through the grouping of mobile instant messages, and we explore how intrinsic and extrinsic motivations affect the adoption of MIM within a group. Based on an empirical structure equation, we propose a computational model to better understand how the intrinsic and extrinsic factors impact on the dynamic evolution of MIM groups' adoption decisions. The model presented in this paper could serve as a foundation on which a vendor can develop different types of marketing strategies. For example, the vendor could develop a product development strategy for a new version of MIM, create different pricing policies for different groups, and so forth. The simulation results indicate that diffusion speed can be increased through an increase in the parameters, and the degree of increase has a significant impact on the diffusion process. Each factor has a significantly different effect on behavioral evolution, and the composition of a group also has a major impact on the diffusion process.

Our study has several potential limitations. First, China has the largest number of mobile phone users, and this study focused on the adoption of mobile instant messaging based on mobile phone usage. However, the findings might be different if the model were examined in another country, where MSN or other messaging tools are in the majority. Secondly, some of the variables in the simulation follow uniform distributions. While these are reasonable assumptions given the absence of other information, multiple different distributions could be used to condition the results. Thirdly, we consider only positive word of mouth in the network, and we do not consider negative spreading, when in fact, when spreading over a network, an agent can spread not only good or positive views to others, but also bad or negative views.

\section{Acknowledgements}

We thank the editor-in- chief, the associate editor, and three anonymous reviewers for their valuable comments and suggestions on this work. This work was partially supported by a grant from the National Natural Science Foundation of China (No. 71101047), China Postdoctoral Science Foundation (No.2011M500119, 
2012T50674), and S\&T project of Hubei Provincial Department of Education (No.D20132201).

\section{References}

1. Iresearch,2012-2013 Annual research report on user behavior of mobile instant messaging (2013) Shanghai: iResearch consulting group.

2. T. Leigh, Agent-based computational economics: modeling economies as complex adaptive systems. Information Sciences, 149(4) (2003) 262-268.

3. S. A. Delre, W. Jager and M. A. Janssen, Diffusion dynamics in small-world networks with heterogeneous consumers. Comput. Math. Organ. Theory, 13(2) (2007) 185-202.

4. N. Gilbert, Agent-based Models (2008). London: SAGE Publications,Inc.

5. M. Fishbein and I. Ajzen, Belief, Attitude, Intention, and Behavior: An Introduction to Theory and Research (1975). MA: Addison-Wesley.

6. I. Ajzen, The Theory of Planned Behavior. Organizational Behavior \& Human Decision Processes, 50(2) (1991) 179211.

7. F. D. Davis, R. P. Bagozzi and P. R. Warshaw, User acceptance of computer technology: a comparison of two theoretical models. Management Science, 35(8) (1989) 982-1003.

8. Y. Lu, T. Zhou and B. Wang, Exploring Chinese users' acceptance of instant messaging using the theory of planned behavior, the technology acceptance model, and the flow theory. Comput. Hum. Behav., 25(1) (2009) 2939.

9. R. K. Garrett and J. N. Danziger, IM = Interruption Management? Instant Messaging and Disruption in the Workplace. Journal of Computer-Mediated Communication, 13(1) (2008) 23-42.

10. A. H. Huang and D. Yen, Usefulness of instant messaging among young users: social versus work perspective. Human Systems Management, 22(2) (2003) 63-72.

11. A. Quan-Haase, J. Cothrel and B. Wellman, Instant Messaging for Collaboration: A Case Study of a HighTech Firm. Journal of Computer-Mediated Communication, 10(4) (2005).

12. Z. Deng, Y. Lu, K. K. Wei and J. Zhang, Understanding customer satisfaction and loyalty: An empirical study of mobile instant messages in China. International Journal of Information Management, 30(4) (2010) 289-300.

13. G. Jiang and W. Deng, An empirical analysis of factors influencing the adoption of Mobile Instant Messaging in China. International Journal of Mobile Communication, 9(6) (2011) 563-583.

14. T. Ma and Y. Nakamori, Agent-based modeling on technological innovation as an evolutionary process.
European Journal of Operational Research, 166(3) (2005) 741-755.

15. K. Bertels and M. Boman, Agent-Based Social Simulation in Markets. Electronic Commerce Research \& Applications, 1(1-2) (2001) 149-158.

16. G. Jiang, B. Hu and Y. Wang, Agent-Based Simulation Approach to Understanding the Interaction between Employee Behavior and Dynamic Tasks. SIMULATION: Transactions of the Society for Modeling and Simulation International, 87(5) (2011) 407-422.

17. T. Weitzel, D. Beimborn and W. Konig, Unified Economic Model of Standard Diffusion: The Impact of Standardization Cost, Network Effects, and Network Topology MIS Quarterly, 30(1) (2006) 489-514.

18. G. Peng, M. Fan and D. Dey, Impact of network effects and diffusion channels on home computer adoption. Decision Support Systems, 51(3) (2011) 384-393.

19. M. E. J. Newman, The Structure and Function of Complex Networks. SIAM Review, 45(2) (2003) 167-256.

20. T. Yu, R. Xiao and X. Gong, Tacit Knowledge Diffusion in Organizational Networks and the Analysis of Learning Strategies. Joumal of Management Sciences, 22(6) (2009) 21-28.

21. M. A. Zaffar, R. L. Kumar and K. Zhao, Diffusion dynamics of open source software: An agent-based computational economics (ACE) approach. Decision Support Systems, 51(3) (2011) 597-608.

22. M. E. Schramm, K. J. Trainor, M. Shanker and M. Y. Hu, An agent-based diffusion model with consumer and brand agents. Decis. Support Syst., 50(1) (2010) 234-242.

23. A.-L. Barabási, Scale-Free Networks: A Decade and Beyond science, 325( 5939) (2009) 412-413

24. C. Luis M.B, On the adoption of innovations with 'network' externalities. Mathematical Social Sciences, 19(3) (1990) 299-308.

25. K.-Y. Lin and H.-P. Lu, Why people use social networking sites: An empirical study integrating network externalities and motivation theory. Computers in Human Behavior, 27(3) (2011) 1152-1161.

26. C. Wang, Y. Hsu and W. Fang, Acceptance of Technology with Network Externalities: An Empirical Study of Internet Instant Messaging Services. Journal of Information Technology Theory and Application (JITTA), 6(4) (2004) 15-28.

27. R. Erhard, Generalization of the Kolmogorov-Smirnov test. Computational Statistics \& Data Analysis, 24(4) (1997) 433-441. 